American Journal of Animal and Veterinary Sciences 5 (2): 139-145, 2010

ISSN 1557-4555

(C) 2010 Science Publications

\title{
Osteoporosis Induction in Animal Model
}

\author{
${ }^{1}$ Kamran Kaveh, ${ }^{1}$ Rashid Ibrahim, ${ }^{2}$ Mohd Zuki AbuBakar and ${ }^{2}$ Tengku Azmi Ibrahim \\ ${ }^{1}$ Department of Clinical Studies, \\ ${ }^{2}$ Department of Preclinical Studies, \\ Faculty of Veterinary Medicine, University of Putra Malaysia, 43400 UPM, \\ Serdang, Selangor, Malaysia
}

\begin{abstract}
Problem statement: Osteoporosis; the major health problem is an imperative disease requires a significant attention to discover different aspects of this threatening abnormality. There are some problems in osteoporotic human population to conduct researches such as lack of a homogenous sample size or ethical problems. So, there was a need to have protocol in animal model to let the scientists discover various feature of this disease to promote health status of the human population. The aim of this research was to make such protocol in animal model. Approach: About 15 rabbits were utilized in the current research. Six animals of Group 1 have undergone bilateral ovariectomy which was followed by IM injection of $1 \mathrm{mg} \mathrm{kg}^{-1}$ day of methylprednisolone. Six rabbits of second group only undergone bilateral ovariectomy and three animal of Group 3 served as a control group. They neither undergone any surgery nor received any medications. After surgery up to four weeks radiographs were taken. At week four animals were euthanized and samples of cortical and chancellors' bone were evaluated by light and scanning electron microscopes. Results: Group 1 completely manifested features of osteoporotic bone. Complete radiolucent view with cortical thinning in radiographs as well as broken, thinned and perforated chancellors bone trabecullae in light and electron microscope confirmed the osteoporotic nature of both cortical and chancellors' bone. Ovariectomy alone did not show sufficient potential to induce osteoporosis in rabbits in such short period. Conclusion: As the conclusion, bilateral ovariectomy following injection of mild doses of glucocorticoids could result in a fast, easy and trustable protocol to induce osteoporosis in rabbit model. This procedure could be utilized in creating osteoporotic rabbits for better understanding of unknown aspects of the recent growing syndrome.
\end{abstract}

Key words: Osteoporosis, glucocorticostroids, bone mass, rabbit

\section{INTRODUCTION}

Osteoporosis is a cluster of abnormalities characterized by a decrease in bone mass and a deterioration in bone microarchitecture which leads to an enhanced fragility of the skeleton and hence to a greater risk of fracture (Jee and Yao, 2001). By improving the public health and aging of the population, the incidence of this disorder is also increasing. Postmenopausal osteoporosis is a major health problem for women society. High susceptibility to fractures in osteoporotic bone, increased osteoclastic resorption and ceased osteoblastic bone formation induce an obligation to know physiopathology, clinical manifestation and treatments of this disease more concisely. Due to slow development of postmenopausal osteoporosis in women and study limitation in human, it is necessary to have a trustable protocol for osteoporosis induction in animal model. Another problem concerned in human society is their life style. Smoking, alcohol consumption and various diet habits inhibit the scientists to have homogenous of society to conduct an investigation. By inducing osteoporotic animal models, the better understanding of osteoporosis, incidence and susceptibility of different bones to fractures and different ways to osteoporotic fractures augmentation would be possible. Animal model also plays an important role in manifesting the unknown aspects of the disease (Turner, 2001).

An appropriate animal model for any research should be based on the following considerations: (1) appropriateness as an analog, (2) transferability of

Corresponding Author: Kamran Kaveh, Department of Clinical Studies, Faculty of Veterinary Medicine,

University of Putra Malaysia 43400 UPM, Serdang, Selangor, Malaysia

Tel: 0060173416818 Fax: 0060389468333 
information, (3) genetic uniformity of organisms where applicable, (4) background knowledge of biological properties, (5) cost and availability, (6) generalizability of the results, (7) ease and adaptability to experimental manipulation, (8) ecological considerations and (9) ethical and societal implications (Turner, 2001; Davidson et al., 1987).

The rabbit is one of the most commonly used animals for medical research, being used in approximately $35 \%$ of musculoskeletal research studies (Neyt et al., 1998). This is in part due to ease of handling and size. The rabbit is also convenient in that it reaches skeletal maturity shortly after sexual maturity at around 5-6 months of age (Gilsanz et al., 1998; Pearce et al., 2007).

Although some differences in bone remodeling between rabbit and human bone, however this laboratory animal is commonly and frequently used in bone tissue researches (Pearce et al., 2007). Since there is a great need to further characterize different aspect of postmenopausal osteoporosis and since rabbit is one of the most common animal in bone researches it is wise to have an osteoporosis induction protocol in this animal model.

So far there are very few and limited data on osteoporosis induction on rabbit model. The main aim of this investigation is to introduce a simple and practical osteoporosis induction protocol in rabbit model.

\section{MATERIALS AND METHODS}

Animals and design: A total of 15 female New Zealand White rabbits, 6 months old with average weight $2.5 \mathrm{~kg}$ were chose for the present study. The protocol for this research project and animal experiment in the current study has been approved by Animal Care and Use Committee of Faculty of Veterinary Medicine, University Putra Malaysia. Rabbits were divided randomly into three groups; group 1 with 6 rabbits had been undergone bilateral ovariectomy and that was followed by IM injections of $1 \mathrm{mg} \mathrm{kg}^{-1}$ day $^{-1}$ methylprednisolone, Group 2 had six rabbits and only undergone ovariectomy and third group with three rabbits served as the control group and did not receive any surgery or medication. The animal of this group were kept under same feeding and environmental condition with other two groups. Rabbits were kept individually in separate cages in room temperature and were fed ad libitum with commercial rabbit pellet provided by a local supplier. They were experiencing $12 \mathrm{~h}$ as days and same hours as night.

Anesthetic protocol: The animals were anesthetized using intramuscular injection of premixed of Ketamine
(100 mL $\left.\mathrm{mL}^{-1} ; 40 \mathrm{mg} \mathrm{kg}^{-1}\right)$, Xylazine $\left(20 \mathrm{~mL}^{-1} ; 5 \mathrm{mg} \mathrm{kg}^{-1}\right)$ and Acepromazine $\left(5 \mathrm{~mL}^{-1} ; 1 \mathrm{mg} \mathrm{kg}^{-1}\right)$. For maintenance, $1 \%$ Isoflurane with oxygen flow rate of $0.6 \mathrm{~L} \mathrm{~min}^{-1}$ (through the face mask) in the completely open circuit using Y piece was utilized.

Surgery protocol: Designated animals have undergone bilateral ovariectomy. For ovariectomy, skin incision was made on the ventral mid line from umbilicus distally for $4 \mathrm{~cm}$. The subcutaneous fascias were incised on the same line. Linea Alba was also stabbed and cut. Left ovary was and three pedicle clamp technique was employed to clamp and ligate the ovarian vessels. Another three clamp technique was used to detach the ovary from uterine horn. The other ovary also was removed in the same manner. For last step, Linea Alba and skin were sutured respectively.

Osteoporosis establishment: Following bilateral ovariectmy, $1 \mathrm{mg} \mathrm{kg}^{-1} \mathrm{day}^{-1}$ of methylprednisolone (Castaneda et al., 2008) was injected intra muscular to the first group. Second group did not receive any medication. Third group neither undergone surgery nor received any medication treatment and served as control group.

Sample examination: The radius and femoral bone were radiographed preoperative, post operative and once a week after surgery up to 4 weeks. At week 4 the rabbits were euthanized by intra cardiac injection of dolethal (Pentobarbital; $200 \mathrm{mg} \mathrm{mL} \mathrm{mL}^{-1}, 2 \mathrm{~mL}$ rabbit) under general anesthesia. The compact as well as spongy bones were removed for histological assessment and Scanning Electron Microscope (SEM) evaluation. Radius was sample of compact and head of femur and wing of ilium were chose as representatives of spongy bones. Statistical evaluation was made by one way ANOVA, of SPSS 16.0 version. $\mathrm{p}<0.05$ was considered as statistically significant.

\section{RESULTS}

Radiographic observation: Radiographs were taken on weekly bases using table top procedure $(44 \mathrm{kVp}$ and $2 \mathrm{mAs}$ ) with an exposure time of $10 \mathrm{~ms}$ and working distance of $1 \mathrm{~m}$ in all groups. At fourth week postoperation, radius in group 1 showed completely radiolucent view with very thin cortex with and abnormal marrow cavity density (Fig. 1a) whereas radius in Group 2 and 3 shows normal bone structure, thick and radiopaque cortex with normal marrow cavity density (Fig. 1b). 


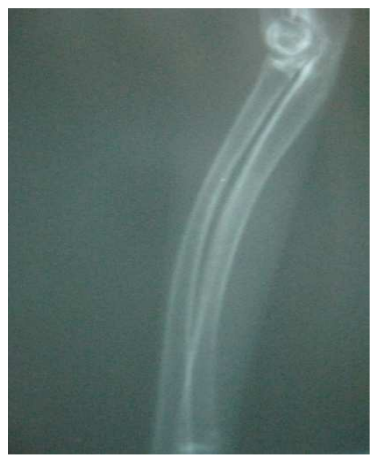

(a)

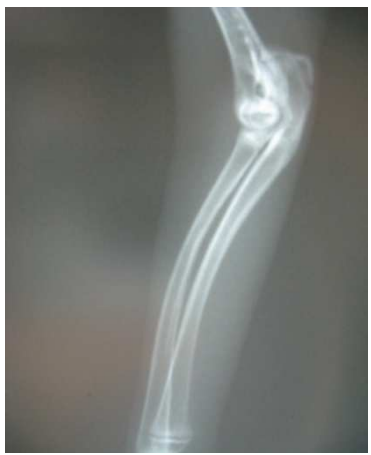

(b)

Fig. 1: At 4th week, Group 1 shows complete feature of osteoporotic bone (a) where as Group 2 and 3 were not qualified to induce osteoporosis (b)

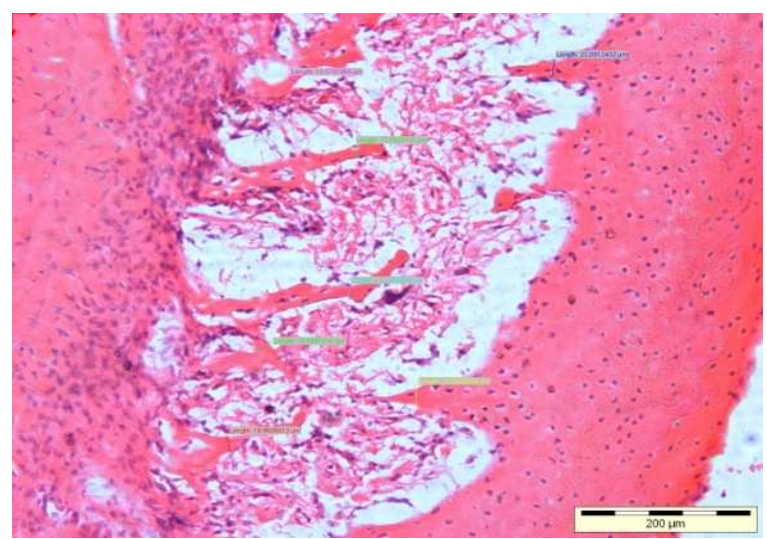

(a)

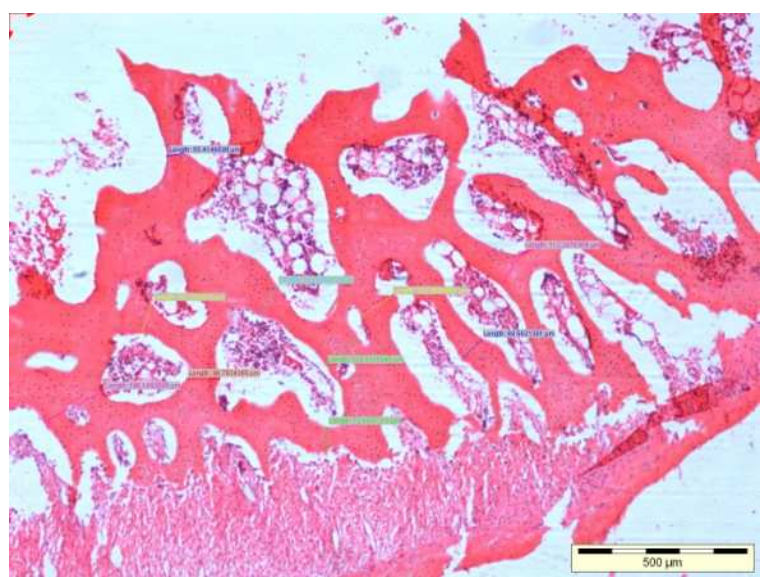

(b)

Fig. 2: At 4th week bone trabeculla shows broken and perforated appearance in Group 1 (a) while showing complete connectivity in other two groups (b) The trabeculla thickness was also measured in all groups

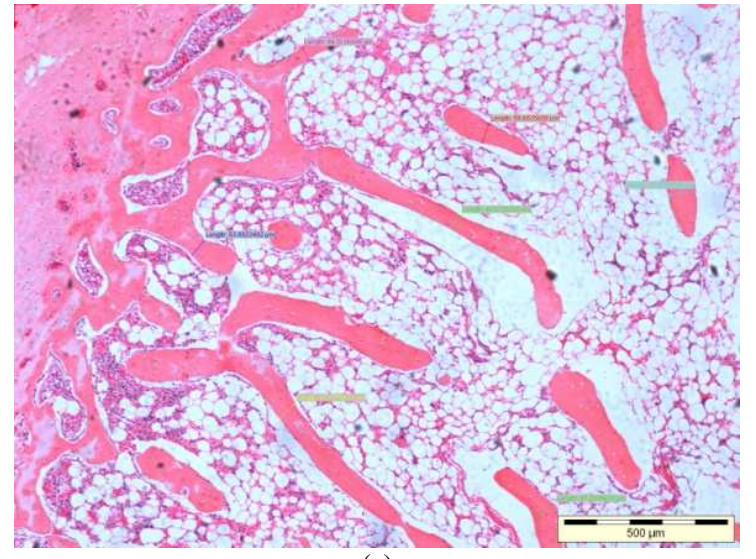

(a)

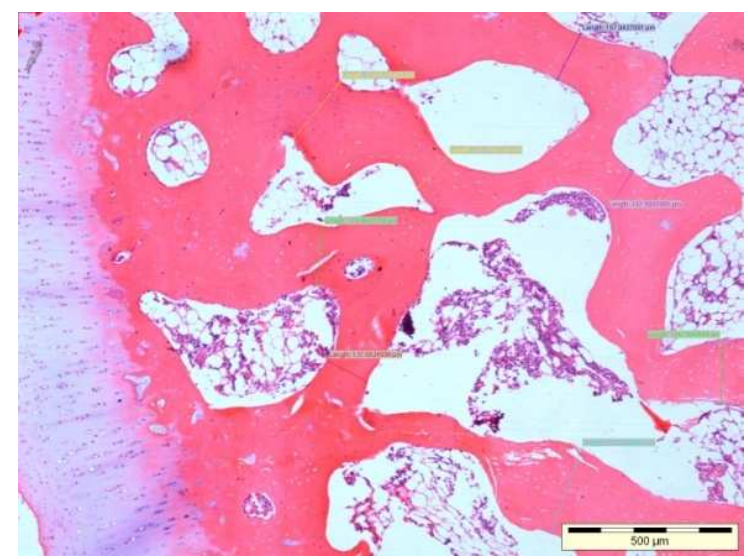

(b)

Fig. 3: Femoral head trabecullae at week 4 shows elongated, thinned and broken trabecullae in Group 1 (a) but it is showing a complete connectivity in other two groups (b). Trabecullae thickness was also measured and compared

Histopathology examination: As the representative of cancellous bone, wing of ilium and head of femur was assessed. In Group 1 in iliac wing, the bone trabecullae was broken and proliferated. There was not continuity in the bone trabecullae in wing of ilium (Fig. 2a). In femoral head, the trabeculae became elongated and broken (Fig. 3a). Second and third groups showed normal, thick bone trabecullae with complete continuity and connectivity in both iliac wing (Fig. 2b) and femoral head (Fig. 3b). Trabecullae thickness was measured in all groups. There was significant difference between Group 1 with Group 2 and $3(\mathrm{p}<0.05)$.

For the cortical bone, size of lacunae in all groups was measured. Group 1 showed larger trabecullae (Fig. 4a) in comparison to the other two groups (Fig. 4b). There was significant difference in lacunar size between Group 1 with Group 2 and 3 ( $p<0.05$ ). 
American J. Animal \& Vet. Sci., 5 (2): 139-145, 2010

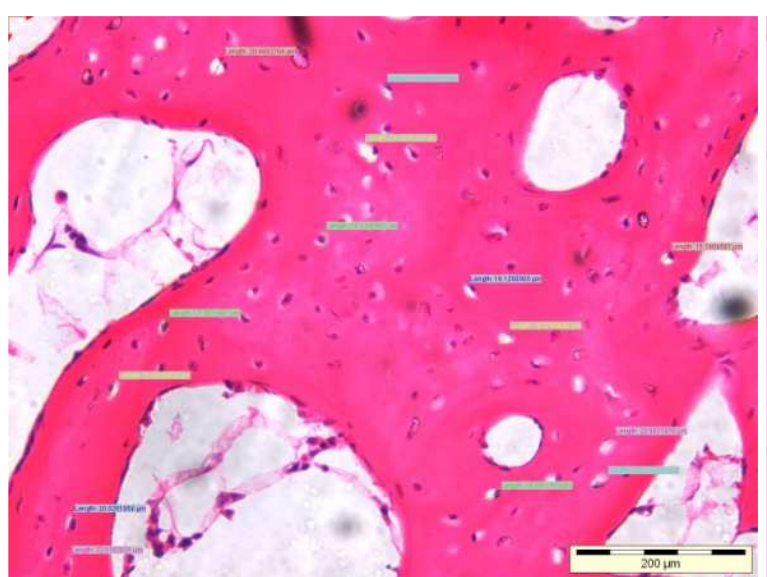

(a)

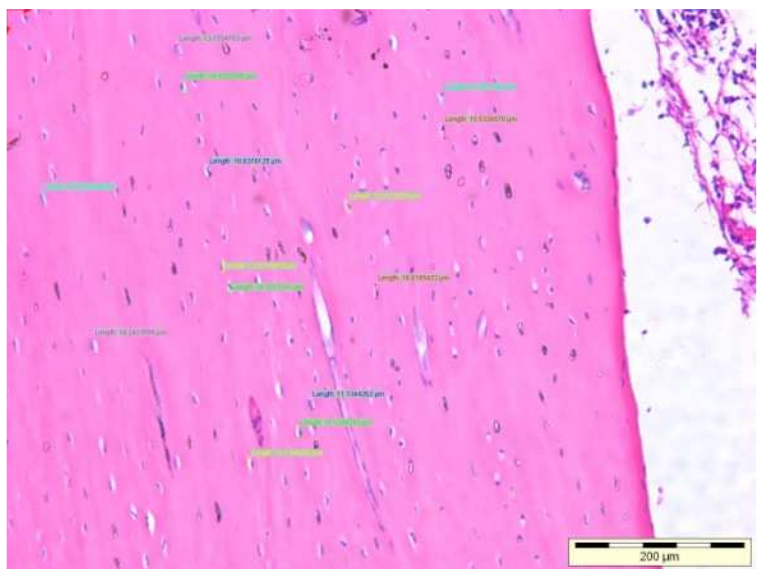

(b)

Fig. 4: Lacunar size in all groups was measured. Osteoporotic bone (a) showed larger size compared to the normal bone (b)

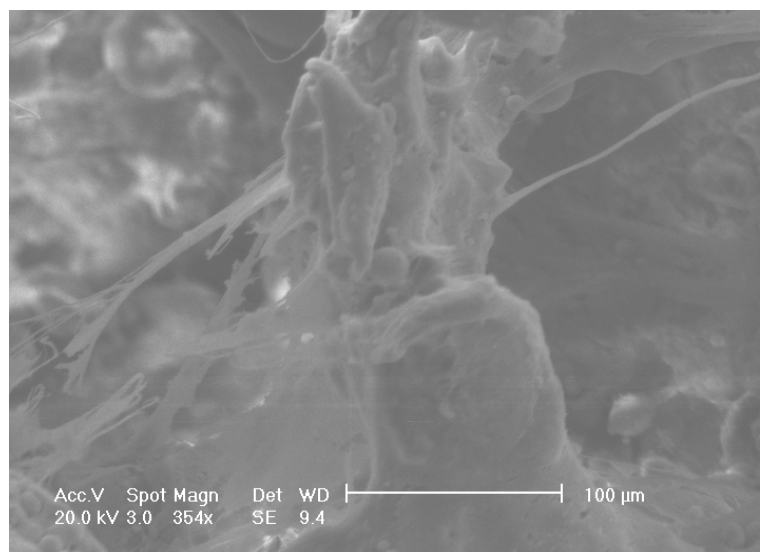

(a)

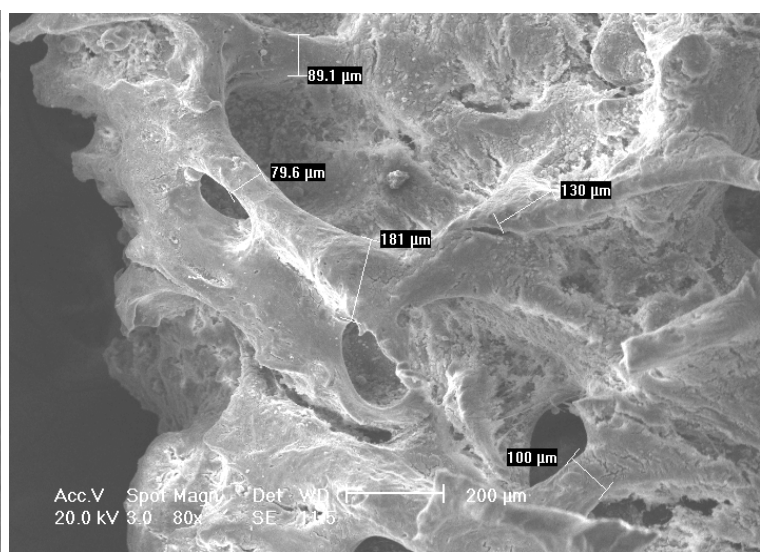

(b)

Fig. 5: Scanning electron microscope shows perforated (arrow), thin and broken treabecullae in osteoporotic animals of Group 1a while other two groups shows (a) complete normal and connected trabecullae (b) Thickness of trabecullae were measured and compared between all groups

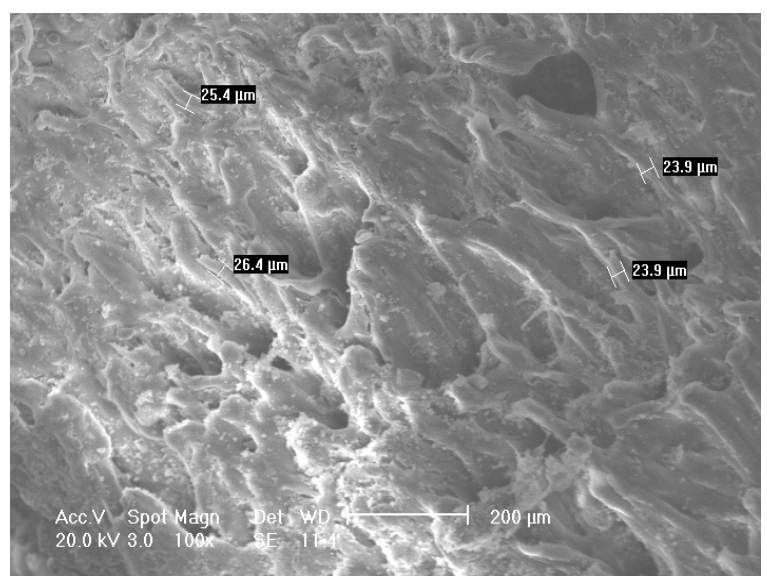

(a)

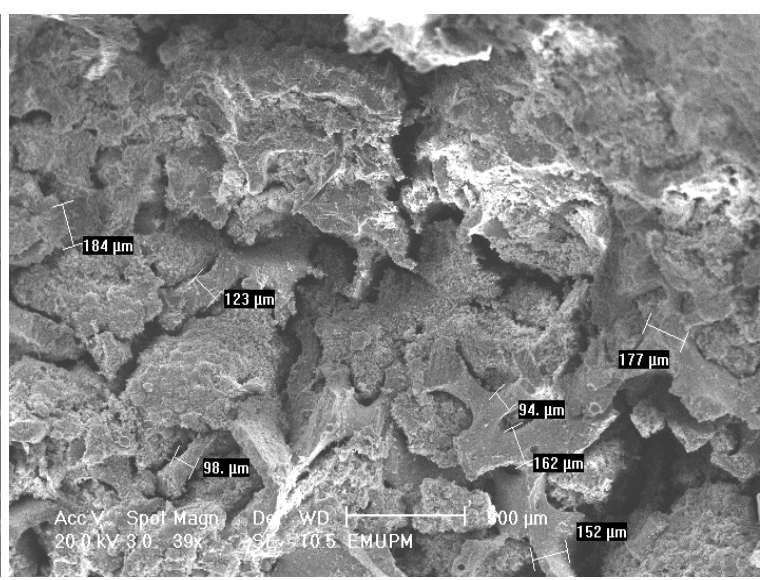

(b)

Fig. 6: Head of femur under SEM, showed thinner trabecullae in Group 1 (a) in comparison with other 2 groups (b) 
Bone thickness was also measured. There was significant difference in bone thickness between Group 1 with Group 2 and $3 \quad(\mathrm{p}<0.05)$. Bone thickness was significantly decreased in the first group.

Scanning electron microscope evaluation: On iliac wing, trabecullae were thinned, broken and perforated. Iliac wing trabecullae thickness was measured in all groups. There was significant difference in trabecullae thickness between Group 1 (Fig. 5a) with Group 2 and 3 (Fig. 5b) (p<0.05).

Femoral head trabecullae thickness was measured by SEM in all groups. Group 1 showed thinner bone trabecullae (Fig. 6a) compared with Group 2 and 3 (Fig. 6b) There was significant difference between normal and osteoporotic trabecullae thickness $(\mathrm{p}<0.05)$.

\section{DISCUSSION}

The result of our study gives the impression to indicate that only group one could successfully achieve in inducing osteoporosis in rabbit model. Group 2 and 3 were completely unsuccessful in osteoporotic animal induction, which means ovariectomy alone was not sufficient in osteoporosis induction.

Radiographic observation showed a complete osteoporotic feature in Group 1. Cortical thinning as well as complete radiolucent view confirmed the osteoporosis by radiologic findings. Histopathologic findings proof the issue by demonstrating broken, perforated and thinned trabecullae in iliac wing and elongated and broken cancellous trabecullae in head of femore. SEM evaluation was in agreement with other two findings. Perforated trabecullae is completely obvious in iliac wing and thinned and broken trabecullae was significantly manifested. All findings agreed that ovariectomy alone is not sufficient in osteoporosis induction and it should be accompanied by injection of a glucocorticoid agent. These findings are in complete agreement with findings of Castaneda et al. (2008).

Studies examining the fracture healing process in osteoporotic bone are very limited and scant which might be due to lack of a standard protocol for osteoporosis induction. In a glance osteoporosis would result in bone fragility and an increase in susceptibility to fracture (Namkung-Matthai et al., 2001). As a health-care problem, osteoporosis now comprises a large percentage of both public and private health spending and is expected to escalate rapidly in the 21 st century, with projected costs of hip fractures alone reaching US\$ 131 billion worldwide by 2050 (Namkung-Matthai et al., 2001). It is a major health problem affecting more than 25 million individuals only in the United States and is responsible for well over one million bone fractures each year (Percival, 1999). The annual financial costs of osteoporosis in the US alone -based on hospitalization costs and acute and long-term care-are estimated to exceed ten billion dollars (Percival, 1999) and with the rapid aging of the population, these costs are expected to increase dramatically. The bone loss that precedes the osteoporotic fracture is a symptomless process, so osteoporosis may go undetected until bones become so brittle that even the slightest trauma would cause a fracture (Dempster and Lindsay, 1993). Spine is the most common region of fracture, with one third of women 65 years and older having sustained spinal vertebrae fractures, leading to loss of height, kyphosis and chronic back pain (Dempster and Lindsay, 1993; Percival, 1999). By extreme old age, one of every three women and one of every six men will have had a hip fracture which, by any measure, is the most devastating of all osteoporotic fractures (Percival, 1999).

With this threatening awaking awareness still little emphasis has been given to the study of fracture healing in osteoporotic bone (Namkung-Matthai et al., 2001). Hence, a trustable osteoporotic inducible protocol in animal model now is showing to be so vital to provide us more information about different aspects of bone fracture and bone healing pattern in this disease.

Bone biopsies performed in osteoporotic women have demonstrated decreased bone formation rates due to diminished osteoblastic function and prolonged remodeling periods (Marie et al., 1989). Microdamages and trabecular microfractures due to abnormalities in trabecular microarchitecture have been seen with aging and osteoporosis (Namkung-Matthai et al., 2001). Alterations in fracture healing with delayed osseous differentiation has also been noted in osteoporotic bone in humans (Fujimoto et al., 2001) due to compositional differences in local growth factors such as transforming growth factor, bone morphogenetic protein (NamkungMatthai et al., 2001) and insulin-like growth factor (Namkung-Matthai et al., 2001). As the consequence, Estrogen deficiency is one of the most important factors in the pathogenesis of osteoporosis. Osteoporotic changes due to estrogen deficiency have been associated with an imbalance between bone formation and bone resorption (Namkung-Matthai et al., 2001).

There are two points of view on the mechanism of the effect of estrogen deficiency on bone union and new bone structure. Cesnjaj et al. (1991) investigated the osteoinductive effects of oestrogen and determined that osteogenesis was decreased and chondrogenesis was retarded in ovariectomized rats. The authors explained 
this finding by hypothesizing that oestrogen deficiency altered the production of osteoinductive proteins such as osteogenin and bone morphogenetic protein, thus disturbing bone matrix formation. This mechanism accounts for the effect of oestrogen deficiency on the early phase of bone union (Cesnjaj et al., 1991). According to a different view, in addition to the negative effects on new bone formation, increased bone turnover will lead to osteoporosis in the new bone as well as in the host bone and thus the new bone will be less sound. Kubo et al. (1999) assert that this mechanism adversely affects the late phase of bone union (Kubo et al., 1999). Walsh et al. (1997) have determined that new bone is less biomechanically sounds in ovariectomized rats, but that is in contrast with Kubo's findings, who revealed that bone structure is less affected in the late phase (Walsh et al., 1997). Arsalan et al. (2003) reported a less compact structure in the callus formation in the periosteal region, inhibition of matrix calcification and a less mature trabecular appearance in bone union (Arsalan et al., 2003).

Histomorphometric analysis of osteoporosis associated with menopause, a natural cause of oestrogen deficiency, has shown that bone resorption and formation are relatively normal in most osteoporotic patients (Arsalan et al., 2003). However, there are subgroups of patients in whom bone resorption is high and formation is low. These heterogeneous findings are explained by the hypothesis that there may be different phases in osteoporosis associated with oestrogen deficiency and that osteoblastic and osteoclastic activity may vary from phase to phase. Higher increases in osteoblastic and osteoclastic activity in the both early and late phases of oestrogen deficiency have been reported. According to Arsalan et al. (2003) finding's increase in activity in the late metabolic phase was higher the early phase of oestrogen deficiency. They have also observed thin, irregular structures in the callus tissues as well as mature trabecullae of osteoporotic rabbits (Arsalan et al., 2003).

Iliac crest biopsies from patients with bone loss caused by estrogen deficiency show reductions in trabecular bone volume, trabecular number and connectivity and these are associated with an increase fracture risk (Lane et al., 2006). These alterations in bone remodeling include an increase in bone resorption and suppression of bone formation such that reductions in trabecular bone mass and architecture occur, including reductions in trabecular thickness and trabecular number (Lane et al., 2006; Dempster, 1989). Also changes in mechanical integrity surrounding osteocytes along with increased lacunar size are observed in osteoporosis (Lane et al., 2006). Lane et al.
(2006) in their study observed changes in elastic modulus surrounding osteocyte lacunae, reduced mineral to matrix ratios in the same areas and an increase in lacunar size. This finding suggests that osteoporosis may alter the metabolism and function of the osteocyte and is not just inducing cell death (Lane et al., 2006). It has been hypothesized that the osteocyte can remodel its local environment, which includes both the lacunae and canaliculi (Baylink and Wergedal, 1971). Baylink and Wergedal (1971) found that osteocyte lacunae have been shown to uptake some materials such as tetracycline, called "periosteocytic perilacunar", indicating their ability to calcify or form bone. Alternatively, these investigators also found acid phosphatase-positive osteocytes near endosteal osteoclastic reserving surfaces, suggesting potential capacity to resorb. They also found greater solubility of the intralacunar mineral surrounding the normal osteocyte (Baylink and Wergedal, 1971). These observations suggest that the osteocyte can both add and remove mineral and as a result, canalicular size increased is another finding of osteoporotic bone.

The results of this study were in agreement with Castaneda et al. (2008) that revealed performing ovariectomy alone is insufficient to induce osteoporosis. Lane et al. (2006) stated the osteocyte-lacunar size would increase in glucocorticoid induced osteoporosis such as finding of the current research and last but not least as Arsalan et al. (2003) reported the trabecullae of the spongy bone would become thinner and broken exactly such as our observation in this research.

\section{CONCLUSION}

In conclusion, osteoporosis is a major increasing population health problem. So there was a necessity to have protocol of animal model of osteoporosis to let the scientist discover more aspects of this abnormality. The outcome of the current investigation was a simple, fast and trustable protocol to induce osteoporosis in rabbit model. Performance of bilateral ovariectomy following $1 \mathrm{mg} \mathrm{kg}^{-1} \mathrm{day}^{-1} \mathrm{IM}$ injection of methylprednisolone seems to be an easy, fast and efficient technique to induce osteoporosis in rabbits.

\section{ACKNOWLEDGEMENT}

Different supports of faculty of veterinary medicine, university Putra Malaysia are greatly acknowledged by the authors. 


\section{REFERENCES}

Arsalan, H., A. Ketani, A. Gezici, A. Kapukaya and S. Necmioglu et al., 2003. The effects of osteoporosis on distraction osteogenesis: An experimental study in an ovariectomized rabbit model. Acta Orthp. Belg., 69: 67-73. PMID: 12666293

Baylink, D.J. and J.E. Wergedal, 1971. Bone formation by osteocytes. Am. J. Physiol., 221: 669-678. PMID: 5570322

Castaneda, S., E. Calvo, R. Largo, R. Gonzalez-Gonzalez and C. de la Pierda et al., 2008. Characterization of a new experimental model of osteoporosis in rabbits. J. Bone Miner. Metabolism., 26: 53-59. DOI: 10.1007/s00774-007-0797-1

Cesnjaj, M., A. Stavljenic and S. Vukicevic, 1991. Decreased osteoinductive potential of bone matrix from ovariectomized rats. Acta. Orthop. Scand., 62: 471-475 PMID: 1950495

Davidson, M.K., J.R. Lindsey and J.K. Davis, 1987. Requirements and selection of an animal model. Isr. J. Med. Sci., 23: 551-555. PMID: 3312096

Dempster, D., 1989. Iliac crest biopsy: Bone histomorphometry in glucocorticoid-induced osteoporosis. J. Bone. Miner. Res., 4: 137-141. DOI: $10.1002 /$ jbmr.5650040202

Dempster, D.W. and R. Lindsay, 1993. Pathogenesis of osteoporosis. Lancet, 341: 797-801. PMID: 8096008

Fujimoto, T., R. Kitazawa, S. Maeda, K. Mizuno and S. Kitazawa et al., 2001. BMP-3 mRNA expression during endochondral ossification of mouse bone tissue. Acta Histochem. Cytochem., 34: $\quad 1-7 . \quad$ http://sciencelinks.jp/jeast/article/200204/000020020401A0544722.php

Gilsanz, V., T.F. Roe, D.T. Gibbens, E.E. Schulz and M.E. Carlson et al., 1998. Effect of sex steroids on peak bone density of growing rabbits. Am. J. Physiol. Endocrinol. Metab., 255: E416-E421. http://ajpendo.physiology.org/cgi/content/abstract/ 255/4/E416

Jee, W.S.S. and W. Yao, 2001. Overview: Animal models of osteopenia and osteoporosis. J. Musculoskel. Neuron. Interact., 1: 193-207. PMID: 15758493
Kubo, T., T. Shiga, J. Hashimato, M. Yoshioka and H. Honjo et al., 1999. Osteoporosis influences the late period of fracture healing in a rat model prepared by ovariectomy and low calcium diet. J. Steroid. Biochem. Mol. Biol., 68: 197-202. PMID: 10416834

Lane, N.E., W. Yao, M. Balooch, R.K Nalla and G. Balooch et al., 2006. Glucocorticoid-Treated mice have localized changes in trabecular bone material properties and osteocyte lacunar size that are not observed in Placebo-treated or estrogendeficient mice. J. Bone. Miner. Res., 21: 466-476. PMID: 16491295

Marie, P.J., A. Sabbagh, M.C. de Vernjoul and A. Lomri, 1989. Osteocalcin and deoxyribonucleic acid synthesis in vitro and histomorphometric indices of bone formation in postmenopausal osteoporosis. J. Clin. Endocrinol. Metab., 69: 272-279. PMID: 2787799

Namkung-Matthai, H., R. Appleyard, J. Jansen, J. Haolin and S. Maastricht et al., 2001. Osteoporosis influences the early period of fracture healing in a rat osteoporotic model. Bone, 28: 80-86. PMID: 11165946

Neyt, J.G., J.A. Buckwalter and N.C. Carroll, 1998. Use of animal models in musculoskeletal research. Iowa Orthop. J., 18: 118-123. PMID: 9807717

Pearce, A.I., R.G. Richards, S. Milz, E. Schneider and S.G. Pearce, 2007. Animal models for implant biomaterial research in bone: a review. Eur. Cell Mater., 13: 1-10. PMID: 17334975

Percival, M. 1999. Bone health and osteoporosis. Applied Nutr. Sci. Rep., 5: 1-6.

Turner, S.A., 2001. Animal models of osteoporosisnecessity and limitations. Eur. Pean Cell Mater., 1: 66-81. http://mobi.ecmjournal.org/journal/papers/vol001/p df/v001a08.pdf

Walsh, W.R., P. Sherman, C.R. Howlett, D.H. Sonnabend and M.G. Ehrlich, 1997. Fracture healing in a rat osteopenia model. Clin. Orthop. Rel. Res., 342: 218-227.

PMID: 9308544 